\title{
Path of sustainable development of agricultural ecosystems in the context of digitalization
}

\author{
Tanzilya Sozaeva ${ }^{1, *}$, Svetlana Gurfova ${ }^{1}$, Dinka Marius-Sorin ${ }^{2}$, and Alfina Pshigosheva ${ }^{1}$ \\ ${ }^{1}$ Kabardino - Balkarian State Agricultural University named after V.M. Kokov, Lenin Avenue, 1V, \\ 360030 Nalchik, Russia \\ ${ }^{2}$ Transylvania University of Braşov, Bulevardul Eroilor 29, 500036 Braşov, Romania
}

\begin{abstract}
Digitalization provides new opportunities for determining the path of sustainable development of agricultural and ecological systems. The article attempts to consider the problems related to the emerging digital divide and the possibility of their leveling accompanying this process both at the international and national levels. Digital transformation is taking place not only in the technology sector and digital companies: its scale extends to all areas of the world economy. It was revealed that digital transformation, accompanied by the development of digital platforms, provides a more complete interaction of agricultural ecosystems with the digital world. Digital platforms have been identified to facilitate the integration of business, social and technological processes that form digital service ecosystems. The main reason for the expansion of the digital segment of agricultural ecosystems is the increase in the transactional sector. The creation of IT platforms based on the digitalization of production and technological processes, jobs; logistics, collaboration of agribusiness entities and public-private partners, consumers, competitors, investors, authorities has been proposed.
\end{abstract}

\section{Introduction}

The problem of digitalization of agricultural ecosystems and sustainable development of rural areas is one of the key issues of modern world science. Digital transformation is accompanied by the development of digital platforms. In this regard, agricultural ecosystems are collaborating with the digital world. The result is the definition of a new path for sustainable development. However, at the same time, digital gaps arise, the need to overcome them by states, which is becoming a priority area of policy in developing countries. Digitalization is taking place not only in the technology sector and digital companies, its scale extends to all areas of the world economy. The digital segment of agricultural and ecological systems is constantly expanding. The main reason for this is the increase in the transactional sector, which accounts for more than $70 \%$ of the national GDP of developed countries. A diversified economy presupposes the presence of large volumes of unique data circulating both within the country and abroad, as a result of which

* Corresponding author: sozaytanzilya@yandex.ru 
information traffic in the national economy grows. It should be noted the higher efficiency of the digital economy in the market, where there are a larger number of participants and there is a high level of penetration of information and communication technologies (ICT).

\section{Materials and Methods}

The materials were the works of foreign and domestic scientists in the field of the theory of agricultural ecosystem management, statistical data of the Federal State Statistics Service, reports of the Ministry of Agriculture of the Russian Federation, as well as reports of the $\mathrm{UN}, \mathrm{FAO}, \mathrm{OECD}$. The system analysis and synthesis were used as the main research methods, which made it possible to assess the current state of the use of ICT and identify the main directions in solving digitalization problems.

\section{Results and Discussion}

In the context of digital globalization, there is a need for a positive synergistic effect on the sustainable development of agricultural ecosystems. Digital transformation of agriculture is part of the nationwide task of the integrated development of agricultural territories. In this regard, it is necessary to locate and specialize in agricultural and industrial production based on a multi-level integrated information space and modern digital technologies. Digital transformation is driven by four trends: mobile technologies, business intelligence, cloud computing, and social media. The entire agri-food chain and its links will change under the influence of digitalization. It will be possible to manage the resources of any element of the system using optimization, an individual approach, rationality and predictability. The system will operate in real time through hyper-connectivity, data-driven. Value chains will be characterized by the coordination of optimal management models for agricultural land, plants and animals. The result of the functioning of digital agriculture will be the creation of efficient agri-food systems adapted to climate change. Furthermore, the level of food security, profitability and stability of enterprises and organizations of the agricultural and industrial complex (AIC) will increase.

Note, that the introduction of digitalization in various spheres of society causes heated disputes among scientists. Thus, the philosophical understanding of this process is today one of the most relevant areas of study of the problems and prospects of IT technologies, considered in the context of NBIC convergence $[1,12,13]$.

Digitalization processes are based on the formation of infrastructure, ecosystem and culture of ICT users. The agricultural and industrial complex is positioned as one of the most dynamic and promising points of ICT application. Thus, in 2018, the volume of exports of services using digital technologies amounted to $50 \%$ of world exports of services, and the size of the digital economy, according to estimates, was in the range of $4.5-15.5 \%$ of global GDP. Nearly $40 \%$ of the value added in the global ICT sector comes from the United States and China. These two countries account for $75 \%$ of patents with blockchain technology, $50 \%$ for the Internet of Things, over $75 \%$ of the cloud computing market, and $90 \%$ of the market value of the largest 70 digital platforms [2,3].

The domestic market of digital technologies in the agricultural and industrial complex in 2020 is estimated at RUB 360 billion, and by 2026 it should grow 5 times. In 2019, the highest costs for the implementation and use of digital technologies in the Russian Federation were observed in the Central Federal District (RUB 1,717,059.7 million). (Table 1). 
Table 1. Costs for the implementation and use of digital technologies in 2019, RUB million.

\begin{tabular}{|c|c|c|c|}
\hline \multirow[b]{2}{*}{$\begin{array}{c}\text { Russian Federation and its constituent } \\
\text { entities }\end{array}$} & \multirow[b]{2}{*}{ Total } & \multicolumn{2}{|c|}{ Including } \\
\hline & & $\begin{array}{l}\text { Internal } \\
\text { expenses }\end{array}$ & $\begin{array}{l}\text { External } \\
\text { expenses }\end{array}$ \\
\hline Russian Federation (RF) & 2316831.4 & 1828652.5 & 488178.9 \\
\hline Central Federal District (CFD) & 1717059.7 & 1365467.4 & 351592.3 \\
\hline Northwestern Federal District (NWFD) & 127356.0 & 95509.9 & 31846.1 \\
\hline Southern Federal District (SFD) & 46607.0 & 35725.8 & 10881.2 \\
\hline North Caucasian Federal District (NCFD) & 13802.3 & 12405.5 & 1396.8 \\
\hline Volga Federal District (VFD) & 176901.6 & 141721.1 & 35180.5 \\
\hline Ural Federal District (UFD) & 106103.4 & 76775.0 & 29328.4 \\
\hline Siberian Federal District (SFD) & 81955.8 & 62394.1 & 19561.7 \\
\hline Far Eastern Federal District (FEFD) & 47045.6 & 38653.7 & 8391.9 \\
\hline
\end{tabular}

The source: [7]

The influence of social and economic transformations and geopolitical factors on the agricultural and industrial complex led to an increase in the indicators of the agricultural sector of the domestic economy, which was not a high-tech industry and did not show a significant increase in labor performance and efficiency. Digital transformation is most in demand today in crop production, processing of agricultural products, as well as their collection, storage and logistics.

Since 2010, organizations of the constituent entities of the Russian Federation have seen a significant increase in the use of local area networks and servers, and since 2015 - "cloud" services (Fig. 1-4).

Modern economic development of agricultural territories is impossible without state regulation of digitalization processes. In the Federal Scientific and Technical Program for the Development of Agriculture for 2017-2025 and the National Technological Initiative (NTI), great importance is attached to IT issues in agriculture. This is reflected in the FoodNet and AeroNet Roadmap, the concept of smart agriculture and priority projects totaling up to RUB 3.3 billion. The departmental project "Digital Agriculture" of the Ministry of Agriculture of the Russian Federation contained 5 units that ensure the adoption of intelligent digital solutions in order to increase labor productivity and sustainable development of agricultural territories. The process of digitalization of the Russian economy lags significantly behind developed countries. Thus, according to AB InBev Efes, the share of IT specialists in the total number of employees in the agricultural sector in the USA, Germany and Great Britain, respectively, is $4.3 ; 4.5$ and $4.1 \%$, while in Russia this indicator is at the level of $2.4 \%$ [4]. 


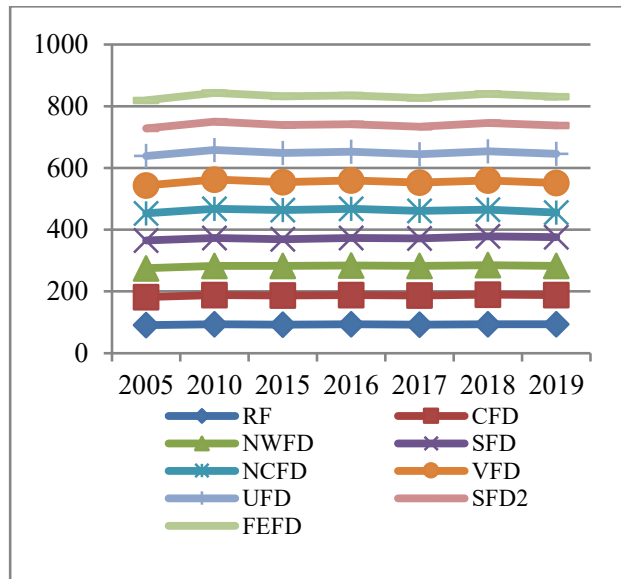

Fig. 1. Personal computers, $\%$ [7].

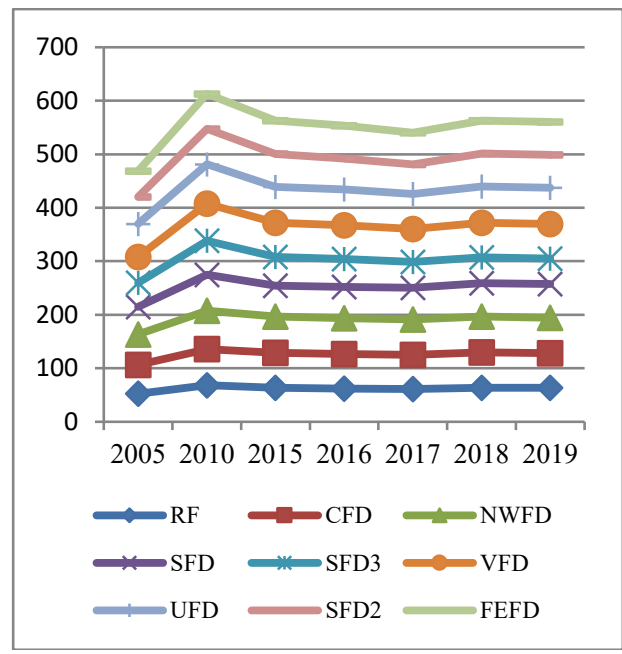

Fig. 3. Local area networks, $\%$ [7].

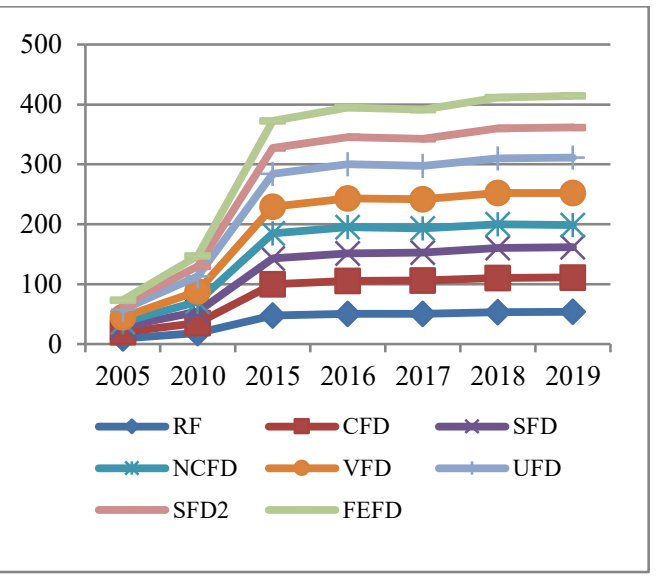

Fig. 2. Servers, $\%$ [7].

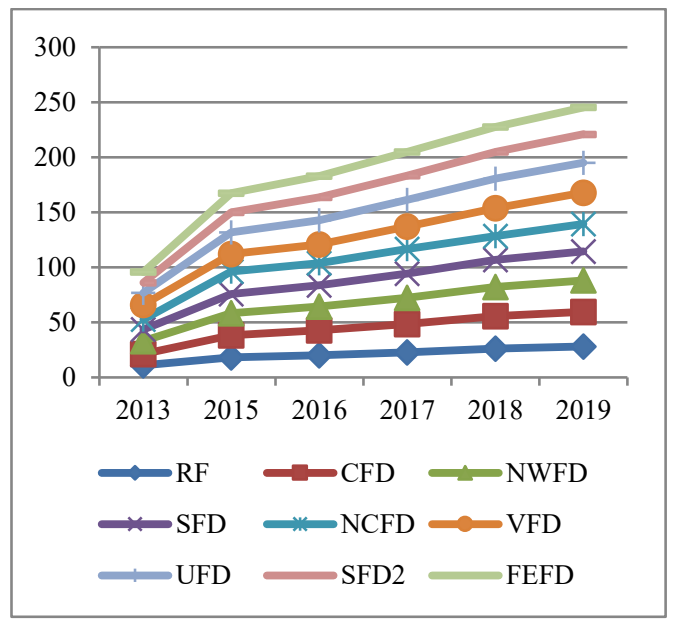

Fig. 4. Cloud services, $\%$ [7].

In the context of defining a path for sustainable development, digital agriculture can provide economic, social and cultural, as well as environmental benefits: the former - by increasing productivity, spending money efficiently and wisely using market opportunities; the latter - by expanding communication and increasing inclusiveness; still others - by optimizing the use of resources and adapting to changing climatic conditions.

The digitalization process has led to a change in the social and economic paradigm of social development. Digital transformation using advanced IT technologies has reshaped the picture of competition, blurred boundaries, changed business models $[5,10,15]$. To unleash the full potential of agricultural digitalization, governments should consider creating and developing an enabling regulatory environment. A high level of administrative capacity is required to develop and effectively manage e-Government projects. In closing the digital divide, governments must initiate the participation of farmers, investors in the agri-food sector, and encourage start-ups to digitize smallholder farmers.

Reaping the potential benefits that are expected from the digitalization of agricultural ecosystems, in full, presupposes a holistic, systems approach. In this regard, the agricultural production systems, the rural economy and the management of natural resources must 
change dramatically. The formation of the digital economy in the agricultural sector is aimed at the transparency of the business environment, attracting investment in the development of digital literacy, the introduction of IT technologies in the agricultural and industrial complex, expanding the relationship of stakeholders in the digital ecosystem, including the public and private sectors, production and the scientific and educational community [8]. Thus, in 2020, as part of the digitalization of Russian agriculture, a single agricultural ecosystem was created: the strategic partnership Digital Agro (Uralchem subsidiary), Agrosignal and Cognitive Pilot, which are part of the SberX ecosystem. The partnership was formed to create a unique product that will combine full cycle agro-ERP and unmanned vehicle management technologies. The expected result is a software and hardware complex that will be able to control equipment, collect, process and transform telemetry data in an unmanned mode, as well as conduct all economic activities of the organization using the capabilities of a single digital field. The total volume of products, services and facilities sold by the parties to the agreement will amount to up to RUB 20 billion per year by 2023 It is planned to introduce these technologies in $30 \%$ of the agricultural and industrial complex market in the amount of over 20 million hectares. The agricultural ecosystem includes a number of elements. In particular, we are talking about the interface; algorithms by which Big Data is analyzed and processed; mobile applications for users from Digital Agro; Cognitive Agro Pilot software and hardware complex, a system that allows autonomous control of agricultural machinery using artificial intelligence (AI); the digital platform "Agrosignal", which is the core of the "Digital Agro" ecosystem (it will serve a base for three technologies integrated into a single agro-ERP). The agricultural ecosystem assumes the possibility of solving all the practical problems facing the economy in one interface; application of accumulated agronomic knowledge and modern technologies in the field of soil science; rational use of equipment and increased control over the implementation of agricultural work. The users can control crop rotation using historical data, constantly updated information from sensors installed on agricultural machinery, knowledge of the soil and climate in their farm, using the Digital Agro application on a tablet. With the help of numbers, you can control all processes and data, set precise tasks for machine operators and monitor their implementation in real time.

Digital transformation of processes on farms will help reduce risks and increase the potential yield of agricultural crops during seasonal work, optimally reduce their cost as a result of reducing losses of a non-production and production nature. The services and solutions of Digital Agro are focused on reducing costs up to $20 \%$, thanks to the consolidated perception of information about the farm and the ability to manage it in real time. As a result of the application of Cognitive Agro Pilot technologies, it is expected to reduce crop losses by $3 \%$ and increase the harvesting speed by $25 \%$. The introduction of all technologies of the agricultural ecosystem will provide a cumulative effect in the form of an additional reduction by an average of $13 \%$ in direct and indirect costs.

Three problems are identified that hinder the development of the digital transformation of agriculture in Russia. The first is the lack of competencies possessed by owners and managers of agricultural formations; the second is in an extremely small number of organizations that could actually do something in this direction; the third is that, as a rule, many proposals are unsuitable for the majority of small and medium-sized farmers that produce most of all agricultural products.

In this regard, solving the problem of digitalization of agriculture requires taking into account a number of factors:

1) lack of systemic and official data, especially their digitalization in urban and rural areas; incomplete information on government support and regulatory support for digitalization. This prevents understanding of the very concept of digital transformation of agriculture; 
2) significant gap in the introduction of digital technologies into the agricultural sector of developed and developing countries, both in global companies and in local and family farms; the introduction of modern agricultural technologies is determined by the availability of financial resources and the level of education;

3) economies of scale: the larger the enterprise, the easier it is to implement digital technologies. In other words, large farms have advantages over small ones. This leads to the emergence of inequality between them and, therefore, inequality between developed and developing countries.

Thus, the digital inequality of agricultural territories due to different levels of access to digital infrastructure does not allow the use of a single set of measures for regions and agricultural formations.

The priority areas in the field of digitalization are:

- collection of more complete information reflecting the applied digital technologies and the process of digital transformations by regions, including separately urban and rural, and population groups;

- creating sustainable business models that allow small farms to be involved in the digital transformation of agricultural territories;

- development of an index reflecting the development of digital agriculture, taking into account the existence of basic and accompanying conditions for the development of digitalization, as well as the potential impact of the process on the economy, ecology and society.

The "Index of Readiness for Implementation of Digital Technologies in the Agricultural Sector" is considered as a basis. It was developed in 2015 by experts from the FAO Regional Office for Europe and Central Asia. The stages of the implementation of the process of digital transformation of agricultural territories have also been determined.

Digitalization affects the increase in the efficiency of economic processes, the growth of competitive advantages, the reduction of unemployment, the rating of countries in the world markets, the development of digital payment systems and electronic money, contributes to the formation of a synergistic effect of competition in commodity markets [5, $6,8]$. The positive is the possibility of digital technologies in solving problems of performing large-scale operations; growth in labor productivity; providing additional benefits to consumers and increasing participation in social and political life. However, there are also negative consequences, in particular, the suppression of the individual by digital addiction and cybersecurity. Moreover, the benefits of digitalization are uneven due to limited access to the Internet; there is an increase in the polarization of the labor market; the influence of natural monopolies is increasing. In this regard, it is impossible to consider digitalization unequivocally. Positive and negative characteristics in the aggregate suggest multivariance of the final results, caused by changes.

It is important to note that the digitalization of the economy will have a significant impact on the transformation processes of labor migration in the future. It caused a change in the behavior of economic agents: a special social group of people appeared, which is engaged in intellectual work and is not tied to a specific place of residence. Digital globalization has caused transformational processes in the migration space and labor relations, including by increasing special interest in highly qualified workers employed in promising sectors of the economy. As a result, the share of highly qualified specialists in the structure of international migration has grown significantly.

To implement digital transformations with the required level and scale, especially in agriculture with its industry specificity, the following conditions must be met:

- fact of the existence of the technologies themselves, characterized by a certain quality, sufficiency of quantity, readiness to work (connectedness), availability, including financial, is the same for all users - both legal entities and individuals; 
- formation of the ecosystem of the digital economy, by which we mean a set of economic agents and conditions ("habitat"), with the possibility of their effective functioning, thanks to the comprehensive connections between them, on the basis of which they interact with each other, exchange resources and transform them, carry out relationships with each other;

- available infrastructure combined with its efficient use;

- corresponding "digital" culture. To fulfill their needs and interests, the users must have basic digital skills, be digitally literate and competent.

Obviously, the place of residence (type of settlement), a city or a village, has a significant impact on the digitalization process as a whole, including due to the "cumulative effect" of factors such as income level, education level, lack of infrastructure, remoteness from communications, etc. Therefore, the digital skills of the urban population when working, e.g., with a word processor and spreadsheets, as well as when transferring files from a computer to peripheral devices and vice versa, are $10-15 \%$ higher than that of rural users. Such conclusions are valid for almost all indicators reflecting the digitalization of agriculture. A relative exception is the electronic display of state and municipal services to the public. Here the gap between urban and rural areas is reduced to about $10 \%$ [9].

The main determinant of economic development, both at the national and regional levels, is the use of digital technologies in order to reduce production costs, meet the needs of the market, since the volume of information is growing squared faster than the volume of production $[10,11,14]$. The priority task of the digital transformation of the agricultural economics is the integration of objective information flows of agricultural producers and government data into the digital agriculture platform in order to provide accurate recommendations to market participants, using artificial intelligence and activating innovative processes. Pilot projects will ensure, through big data, the digital transformation of the agricultural sector of the economy with the formation of digital platforms. Currently, only $10-15 \%$ of Russian farmers use digital platforms. With regard to drones and sensors that allow collecting information about the weather, controlling the operation of equipment, monitoring crops and other key agricultural processes, the share of coverage is higher here, and, therefore, the picture looks more optimistic.

\section{Conclusions}

It is important to note that it is necessary to create IT platforms based on the digitalization of production and technological processes, jobs; logistics, collaboration of agribusiness entities with public-private partners, consumers, competitors, investors and authorities. The basic requirements for the creation of digital platforms have been developed for the following groups:

1) high availability (in terms of economic entities of the agricultural and industrial complex with advanced technologies);

2) relative availability (in terms of enterprises of agricultural service, food and processing industries, trade, poultry farms, stable and profitable functioning);

3) adaptable (in terms of medium and large agricultural enterprises that have sufficient material, financial and labor resources in order to use modern information technologies);

4) not capable of independent adaptation (we are talking about unprofitable enterprises, small peasant (farmer) and other households).

Regional pilot projects will allow, using big data, to digitize agriculture based on digital technologies, and to activate them in everyday life. It is advisable to develop new tools that allow the state, business and society to interact on the principles of inclusiveness, to expand the participation of society in determining science and technology policy. Moreover, it is very important how the rural population will perceive advanced technological solutions, 
which is closely related to the level of requirements for digital competencies and their dynamics.

The digital economy presupposes a new stage of introducing the achievements of scientific and technological progress in all sectors and spheres of social and economic activity, as well as in the foreign and domestic policy of the state. The created platform solutions for digital agriculture have a goal: to form a modular platform that will be able to globally predict supply and demand. In this case, various types of information will be used: from satellites, soil and climatic, from tax, customs, statistical authorities and others. The information will have established formats and will be provided online. For efficient agriculture, the transition to management based on planning and risk forecasting and economic security, interfaces are required that allow connecting logistics and transport systems and feedback data processing systems ("counter-manufacturer") in order to maximize consumer preferences. Furthermore, the role of non-profit associations (unions) in the scenario of digital transformation of agricultural ecosystems, the implementation of innovative solutions, which will lead to the formation of a private-state digital agricultural and industrial complex management system, is growing.

\section{Acknowledgment}

The study was conducted with the financial support of the Russian Foundation for Basic Research within the scientific project No. 20-010-00838 A.

\section{References}

1. J. Canton, Managing Nano-Bio-Info-Cogno Innovations: Converging Technologies in Society, 33-45 (2006)

2. Digital Economy Report. 2019. UNCTAD (2019)

3. European Commission. 2019. ${ }^{2 \text { nd }}$ Survey of Schools: ICT in Education (2019)

4. S. A. Gurfova, Economics and Entrepreneurship, 3 (116)

5. Indira R. Mikitaeva, Marina T. Tekueva, Mikhail Kh. Balkizov, Tanzilya Kh. Sozaeva, Orgutsel davranis arastirmalari dergisi, 3(2), 189-197 (2018)

6. Kh. M., Rakhaev, T. Kh., Sozaeva, S. Kh. Sulumov, Spatial Polarization of National Economy: Current State and Development Priorities, 50-55 (2019)

7. Regions of Russia. Social and economic indicators, Rosstat (2020)

8. T. Kh. Sozaeva, A. Y. Pshigosheva, A. M. Tramova, T. Kh. Toguzaev, International Journal of Recent Technology and Engineering, 8(3), 8286-8292 (2019)

9. T. H. Sozaeva, A. Yu. Pshigosheva, S.A. Gurfova, I. R. Mikitaeva, Agrarian territories in the context of the formation of a digital economy: problems and prospects (2020)

10. S. A. Tumenova, T. Kh. Sozaeva, Knowledge Economy: Noospheric Context of Development European Proceedings of Social and Behavioural, 434, 3226-3236 (2019)

11. S. A. Tumenova, M. M. Mustaev, F. A. Mambetova, European Proceedings of Social and Behavioural Sciences, 92, 339, 2561-2568 (2020)

12. A. V. Frolov, Innovations, 7(177), 63-73 (2013)

13. J. A. Schumpeter, The Theory of Economic Development (1982)

14. I. L. Litvinenko, Z. S. Zhangorazova, Y. B. Mindlin, R. A. Shichiyakh, N. Y. Veselova, G. V. Petruk, International Journal of Applied Business and Economic Research, 13 (2017) 
15. Kh. Uyanayeva, N. Kokov, T. Toguzayev, M. Kuchukov, F. Alibius, 3rd International Conference on Agribusiness, Environmental Engineering and Biotechnologies, AGRITECH-III 2020, 2 (2020) 